Research Paper

\title{
Downregulation of Ubiquitin-conjugating Enzyme UBE2D3 Promotes Telomere Maintenance and Radioresistance of Eca-109 Human Esophageal Carcinoma Cells
}

\author{
Hui Yang ${ }^{1 *}$, Lin $\mathrm{Wu}^{1,3 *}$, Shaobo $\mathrm{Ke}^{1}$, Wenbo Wang ${ }^{1,2}$, Lei Yang1,2, Xiaojia Gao ${ }^{1}$, Hongyan Fang1, Haijun \\ $\mathrm{Yu}^{1,2}$, Yahua Zhong ${ }^{1,2}$, Conghua Xie ${ }^{1,2}$, Fuxiang Zhou ${ }^{1,2}$, Yunfeng Zhou ${ }^{1,2}{ }^{\bowtie}$ \\ 1. Hubei Key Laboratory of Tumor Biological Behavior, Hubei Cancer Clinical Study Center, Zhongnan Hospital, Wuhan University, Wuhan, China; \\ 2. Department of Radiation Oncology \& Medical Oncology, Zhongnan Hospital, Wuhan University, Wuhan, China; \\ 3. Department of Clinical Oncology, Taihe Hospital, Hubei University of Medicine, Shiyan, China. \\ * These authors contributed equally to this work.
}

$\square$ Corresponding authors: Fuxiang Zhou, MD, PhD, Department of Radiation Oncology \& Medical Oncology, Zhongnan Hospital, Wuhan University, 169 Donghu Road, Wuhan 430071, China. Phone: 86-27-67813155. Email: fxzhouwhu@gmail; Yunfeng Zhou, MD, PhD, Department of Radiation Oncology \& Medical Oncology, Zhongnan Hospital, Wuhan University, 169 Donghu Road, Wuhan 430071, China. Phone: 86-27-67812889. Email: yfzhouwhu@163.com.

( ) Ivyspring International Publisher. Reproduction is permitted for personal, noncommercial use, provided that the article is in whole, unmodified, and properly cited. See http://ivyspring.com/terms for terms and conditions.

Received: 2015.12.18; Accepted: 2016.04.26; Published: 2016.06.06

\begin{abstract}
Ubiquitin-conjugating enzyme UBE2D3 is an important member of the ubiquitin-proteasome pathways. Our previous study showed that the expression of UBE2D3 was negatively related to human telomerase reverse transcriptase (hTERT) and radioresistance in human breast cancer cells. However, in esophageal carcinoma, the exact effects and mechanisms of UBE2D3 in radioresistance remain unclear. This study shows that UBE2D3 knockdown was associated with significant increases in radioresistance to $\mathrm{X}$-rays, telomerase activity, telomere length, and telomere shelterins. UBE2D3 knockdown-mediated radioresistance was related to a decrease in the spontaneous and ionizing radiation-induced apoptosis, resulting from a decrease in the $\mathrm{Bax} / \mathrm{Bcl}-2$ ratio. Furthermore, UBE2D3 downregulation was associated with increased G1-S phase transition and prolonged IR-induced G2/M arrest through over expression of cyclin DI, decrease of CDC25A expression and promotion of the ATM/ATR-Chk1-CDC25C pathway. Moreover, UBE2D3 downregulation reduced spontaneous DNA double-strand breaks and accelerated the repair of DNA damage induced by IR. The current data thus demonstrate that UBE2D3 downregulation enhances radioresistance by increased telomere homeostasis and prolonged IR-induced G2/M arrest, but decreases the IR-induced apoptosis and the number of DNA damage foci. These results suggest that UBE2D3 might be a potential molecular target to improve radiotherapy effects in esophageal carcinoma.
\end{abstract}

Key words: UBE2D3; telomere maintenance; radioresistance; esophageal carcinoma; DNA damage repair.

\section{Introduction}

Radiotherapy is one of the most common treatments for esophageal carcinoma. However, the differences in the intrinsic radioresistance of cancer cells of esophageal carcinoma patients imply that increasing the dose of radiotherapy does not necessarily improve 5-year survival or disease-free survival [1]. Thus, it is important to identify new target proteins to enhance the effectiveness of radiotherapy.

Radioresistance can be regulated by a series of pathways, including DNA damage repair [2], telomere maintenance [3], cell cycle progression [4], 
cell apoptosis [5], and cell proliferation [6]. Some reports have shown that the ubiquitin/proteasome system (UPS) participates in the regulation of these pathways [7]. Thus, UPS family members might be potential targets for enhancing the sensitivity of radiotherapy. Our previous study showed that the ubiquitin conjugating enzyme UBE2D3 was negatively related to radioresistance in breast cancer cells [8]. However, the exact effects and mechanisms of UBE2D3 for radioresistance in esophageal carcinoma remain unclear.

UBE2D3, a member of the UbcH5 family, has attracted increasing attention from biologists due to its roles as the initial ubiquitinating enzyme of key regulatory molecules such as cyclin D1 [9], IkB [10], TP53, and MDM2 [11]. Okamoto et al. detected UBE2D3 mRNA expression in 25 tumor cell lines and 24 normal cell lines and showed that UBE2D3 mRNA expression was significantly lower in tumor cell lines than in normal cell lines [12]. Mittal et al. demonstrated that low expression of UBE2D3 is an important index to evaluate the degree of malignant invasion in breast cancer, on account of its role in suppressing cell cycle progression by downregulating cyclinD1 [9]. Moreover, UBE2D3 increases the function of BRCA1, which not only acts as a tumor suppressor gene but also takes part in DNA damage repair by ubiquitin in a variety of downstream molecules [13]. The deubiquitinating enzyme, Otub1, also plays a crucial role in the suppression of the DNA damage repair signal via inhibition of BRCA1 and H2AX ubiquitination mediated by suppression of the ubiquitin conjugating enzyme E2 family member, UBE2N [14]. UBE2D3 suppresses UBE2N via monoubiquitination of Otub1 at lysine 59 and 109 residues [14-16]. Thus, UBE2D3 may affect cancer progression, cell cycle, cell apoptosis, and DNA damage repair. For instance, our team has found that UBE2D3 is negatively related to human telomerase reverse transcriptase (hTERT) [8] and low expression of UBE2D3 is associated with a relatively poor prognosis in patients with esophageal carcinoma [17].

Therefore, our study was designed to investigate the mechanisms by which UBE2D3 regulates radioresistance in esophageal carcinoma, as well as to elucidate the relationship between UBE2D3 and radioresistance in esophageal carcinoma cells and to explore the function of UBE2D3 in five biological processes: telomere homeostasis, cell proliferation, cell cycle, cell apoptosis, and DNA damage repair.

\section{Methods}

\section{Cell lines, transfection, plasmids and reagents.}

The human esophageal carcinoma cells Eca-109 were acquired from Taihe Hospital, Shiyan, Hubei, China. The cells were cultured in Roswell Park Memorial Institute (RPMI)-1640 medium (Thermo fisher, USA), supplemented with $10 \%$ fetal bovine serum and incubated under $5 \% \mathrm{CO}_{2}$ at $37^{\circ} \mathrm{C}$.

ShRNA duplexes designed against UBE2D3 and the negative control were described previously [8]. The plasmids containing these sequences were named pU6/GFP/Neo-shRNA-UBE2D3 and pU6/GFP/ Neo-shRNA, respectively, bought from Shanghai GenePharma (Shanghai, China), and collected by our team.

Cells were seeded in a 6-well plate culture flasks with $3^{*} 10^{5}$ per well, after $24 \mathrm{~h}$, we used turbofect (Thermo fisher, USA) as a transfection reagent according to the manufacturer's instructions. Twenty hours after transfection, UBE2D3 low-expressing cells and its negative control cells were selected with $600 \mathrm{ug} / \mathrm{ml}$ G418 (Biosharp, China) for 5 weeks. The stable transfection cell lines were named Eca-109-NC and Eca-109-sh, respectively.

\section{Clonogenic assay}

We exchanged the cell medium at $60-70 \%$ confluence, $24 \mathrm{~h}$ prior to clonogenic assay. Next day, Eca-109-NC and Eca-109-sh cells were plated into seven 6-well plates, respectively, with different numbers of $100,100,200,400,800,1,000,2,000$ per one plate. After $24 \mathrm{~h}$, each group was irradiated with graded doses of $0,1,2,4,6,8,10 \mathrm{~Gy}$, respectively, by an X-ray generator (Primus High-Energy Siemens) at a dose rate of $2 \mathrm{~Gy} / \mathrm{min}$. After 14 days of incubation, the colonies were fixed and stained with crystal violet ( $1 \%$ in absolute alcohol). Those colonies containing more than 50 cells were scored as viable colonies. These data were fit into the sing-hit multi-target model, and survival curves for each group were demonstrated using GraphPad prism 5.0 software. Radiobiological parameters, D0, Dq and SF2, were calculated according to the survival curves. Sensitization enhancement ratio (SER) was calculated as the ratio of $\mathrm{D} 0_{(\mathrm{Eca}-109-\mathrm{NC})}$ to $\mathrm{D} 0_{\text {(Eca-109-sh). }}$.

\section{RNA Extraction and Quantitative Real-Time PCR}

Total RNA was isolated from cell lines using TRIzol reagent (Biosharp, China) according to the manufacturer's protocol. cDNA was synthesized from no more than $5 \mu \mathrm{g}$ of total RNA by the RecertAidTM First-Strand cDNA Synthesis Kit (Fermentas, Canada) at $42^{\circ} \mathrm{C}$ for $10 \mathrm{~min}$, followed by $75^{\circ} \mathrm{C}$ for $2 \mathrm{~min}$. Real-time PCR was performed with SYBR Premix Ex $\mathrm{Taq}^{\mathrm{TM}}$ (Takara, Japan) in a $25 \mu \mathrm{L}$ reaction volume $(12.5 \mu \mathrm{L}$ SYBR Premix Ex Taq, 200mM forward and reverse primers, $10 \mu \mathrm{L} \mathrm{H} 2 \mathrm{O}$ and $1.5 \mu \mathrm{L}$ cDNA 
template) on a MJ Opticon Monitor Chromo4TM instrument (Bio-Rad, CA). The human GAPDH gene was performed as an internal control. The following protocol was used for GAPDH and UBE2D3: preincubated at $95^{\circ} \mathrm{C}$ for $30 \mathrm{sec}$ followed by 40 cycles of $95^{\circ} \mathrm{C}$ for $5 \mathrm{sec}, 60^{\circ} \mathrm{C}$ for $15 \mathrm{sec}$ and $72^{\circ} \mathrm{C}$ for $30 \mathrm{sec}$. Sangon Biotech (Shanghai, China) helped us design and synthesize the primers for UBE2D3 and GAPDH as follows: UBE2D3 (forward primer 5'CCGGACCTTTGAGCATACAC-3', reverse primer 5'GCCTTGATATGGGCTGTCAT-3'); GAPDH (forward primer 5'-TGGAAGGACTCATGACCACA-3', reverse primer $5^{\prime}$-TTCAGCTCAGGGATGACCTT-3'). Data were analyzed by the 2-DDCt method.

\section{Western Blot Analysis}

Cultured cells were rinsed twice with pre-cold phosphate-buffered saline (PBS) and mixed with 250 $\mu \mathrm{L}$ lysis buffer (Beyotime biotechnology, China) and 1mM PMSF (Biosharp, China). After $10 \mathrm{~min}$ on the ice, a scraper was used to help transfer the cell lysate into an Eppendorf tube. The cells were ultrasonicated 3 times for $30 \mathrm{sec}$ on the ice and then centrifuged at $12,000 \mathrm{rpm}$ for $10 \mathrm{~min}$, and the supernatant was collected into another Eppendorf tube. A BCA protein assay kit (Beyotime biotechnology, China) was performed to calculate the protein concentration, and then the samples were incubated in boiled water for more than $5 \mathrm{~min}$. These samples were separated by $10 \%$ SDS-PAGE and transferred to PVDF membranes. After blocking with $5 \%$ skim milk in TBST, the membranes were incubated with primary antibodies, against human UBE2D3 (1:1000 dilution; abcam ab176568), $\beta$-actin (1:10000 dilution; TDY TDY051, Beijing, China), hTERT (1:1000 dilution; abcam ab32020), PTOP (1:1000 dilution; abcam ab57595), TRF1 (1:1000 dilution; abcam ab10579), TRF2 (1:1000 dilution; abcam ab13579), POT1 (1:1000 dilution; proteintech 10581-1-AP), TIN2 (1:1000 dilution; abcam ab197894), RAP1 (1:1000 dilution; abcam ab47234), ATM (1:1000 dilution; Cell Signaling Technology \#2873S, Danvers, MA), ATR (1:1000 dilution; Cell Signaling Technology \#2790S, Danvers, MA), p-ATM (1:1000 dilution; Cell Signaling Technology \#2873S, Danvers, MA), p-ATR (1:1000 dilution; Cell Signaling Technology \#2873S, Danvers, MA), үH2Ax (1:1000 dilution; abcam ab37168), Cyclin D1 (1:1000 dilution; bioworld BS1741), CDC25C (1:1000 dilution; BBI), CDC25A (1:1000 dilution; abcam ab991), Chk1 (1:1000 dillution; proteintech 10362-1-AP) at $4{ }^{\circ} \mathrm{C}$ overnight, followed by incubation with Horseradish peroxidaseconjugated secondary antibody diluted at 1:20,000. Specific bands were visualized by ECL (Advansta, USA). Autoradiographs were recorded onto X-ray films (Eastman kodak Co, USA). The ImageJ analysis program was used to analyze the density of bands in the resulting films.

\section{Genome DNA extraction and real-time PCR to determine relative telomere length}

Genomic DNA was extracted from Eca-109-NC and Eca-109-sh cell lines by standard procedures using the E.Z.N.A. Tissure DNA Kit (Omega Bio, China) and stored at $-20^{\circ} \mathrm{C}$. Real-time PCR assay was performed to measure the average telomere length ratio as described by Cawthon [18]. The reaction volume was described as above using the Takara real-time PCR kit (Takara Bio, Japan). The 36B4 (single copy gene) was used as an internal control, The cycling conditions were performed as follows: preincubation for $5 \mathrm{sec}$ at $95^{\circ} \mathrm{C}$, followed by 35 cycles at $95^{\circ} \mathrm{C}$ for $15 \mathrm{sec}$ and $54^{\circ} \mathrm{C}$ for $2 \mathrm{~min}$. The primers of telomere primer and single copy gene primer were synthesized by Sangon Biotech (Shanghai, China) as follows: tel1, 5'-GGTTTTTGAGGGTGAGGGTGAGG GTGAGGGTGAGGGT-3'; tel2, 5'-TCCCGACTATCC СТАТСССТАТСССТАТСССТАТСССТА-3'; 36B4u, 5'-CAGCAAGTGGGAAGGTGTAATCC-3'; 36B4d, 5'-CCCATTCTATCATCAACGGGTACAA-3'. The instrument and the method for data analysis are described as above.

\section{Telomerase activity}

In order to harvest $2 * 10^{5}$ cells in Eca-109-NC and Eca-109-sh cell lines respectively, Invitrogen countess were used to count cell concentration in these two cell lines. The samples were performed for detecting the telomerase activity by using the TeloTAGGG Telomerase PCR ELISA Kit (Roche, Switzerland) as recommended by the manufacturer. Model 550 Microplate Reader (Bio-Rad, USA) was used to measure the absorbance of each reaction products at the wavelength of $450 / 690 \mathrm{~nm}$.

\section{Analysis of cell cycle and apoptosis by flow cytometry}

The cell cycle was assessed in cells without irradiation and cells exposed to $6 \mathrm{~Gy}$ of ionizing radiation, and then cells were incubated for the indicated times. Cells were filed in $70 \%$ ethanol overnight, and then treated with RNase for $20 \mathrm{~min}$ before addition of $5 \mathrm{mg} / \mathrm{ml}$ propidium iodide, and analyzed by flow cytometry (Beckman Coulter, USA). Experiments were performed in triplicate. Apoptosis was performed using an Annexin V-PE Apoptosis Analysis kit (Sungene Bio, China) according to the manufacturer's instructions. Fluorescence was measured using a flow cytometer and the data were analyzed with CellQuest software. All samples were 
assayed in triplicate.

\section{Cell proliferation assay}

Cells diluted with RPMI-1640 medium containing $10 \%$ fetal bovine serum, were seeded at $10^{3}$ cells/well in 96-well plates and cultured in $100 \mu \mathrm{L}$ culture medium. Six identical wells were used for each sample. After $24 \mathrm{~h}, 10 \mu \mathrm{L}$ CCK-8 was added to each well, and the plates were incubated at $37^{\circ} \mathrm{C}$ for 2 h. The absorbance of each well was then read at 450 nm using a 96-well plate reader. Each experiment was performed at least three times in triplicate wells.

\section{Immunofluorescence assay by confocal laser scanning microscopy}

The residual DNA double-strand breaks (DSBs) were examined by immunoflorescence detection of yH2AX foci. Cells grown on coverslips (Fisher Scientific, USA) were divided into 4 groups: Eca-109-NC, Eca-109-sh, IR+Eca-109-NC, IR+Eca-109-sh. Twenty four hours later, cell groups without radiation exposure were fixed in $4 \%$ formaldehyde for $20 \mathrm{~min}$, washed with PBS for $5 \mathrm{~min}$ twice, treated with $0.2 \%$ Triton X-100 for $20 \mathrm{~min}$, blocked in 5\% (w/v) BSA for $2 \mathrm{~h}$ and incubated with antibody to $\mathrm{YH}$ AX (ser139, dilution 1:400; Millipore Corp., USA) overnight at $4^{\circ} \mathrm{C}$. After washing with PBS for $5 \mathrm{~min}$ for three times, the secondary FITC-conjugated antibody (Millipore Corp., USA) was added for $1 \mathrm{~h}$ at $37 \mathrm{C}$, and the slides were washed with PBS and stained with DAPI. At last, images were recorded by a confocal microscope (Leica Microsystems GmbH, Wetzlar, Germany). Each experiment was carried out in triplicate.

\section{Statistical analysis}

All data are expressed as means \pm SD. Student's t-test was used to determine statistical significance at $\mathrm{p}<0.05$. SPSS 19.0 and Graphpad Prism 5 software were used for the statistical analyses.

\section{Results}

\section{Efficient knockdown of UBE2D3 in Eca-109 cells}

Real-time PCR and western blots were used to analyze UBE2D3 expression. A comparison of Eca-109-NC and Eca-109-sh cells showed that the expression of UBE2D3 was reduced in Eca-109-sh cells both at the mRNA (Figure 1A) and protein levels (Figure 1B, 1C) $(\mathrm{P}<0.05)$. However, no apparent change in UBE2D3 expression was observed in Eca-109-NC and Eca-109 cells. These results indicate that the stable transfection with pU6/GFP/Neo-shRNA-UBE2D3 effectively knocked down UBE2D3 expression in Eca-109-sh cells.

\section{UBE2D3 downregulation confers radioresistance}

To investigate the effect of UBE2D downregulation on the cell response to radiation, Eca-109-NC and Eca-109-sh cells were exposed to different doses of radiation and subjected to a clonogenic assay. Cell clones were counted and radiobiological parameters were described by survival curves for each cell type. Compared with the negative control group, the survival fractions of Eca-109-sh cells were much higher at each dose (Figure 2). The calculated radiobiological parameters are shown in Table 1. D0, Dq and SF2 values in the Eca-109-sh group were significantly lower than those of the Eca-109-NC groups ( $\mathrm{P}<0.05)$. Negative control and blank control groups showed no significant change. The SER value was $0.605 \pm 0.102$. These results suggest that UBE2D3 downregulation enhances the radioresistance of Eca-109 cells.
A

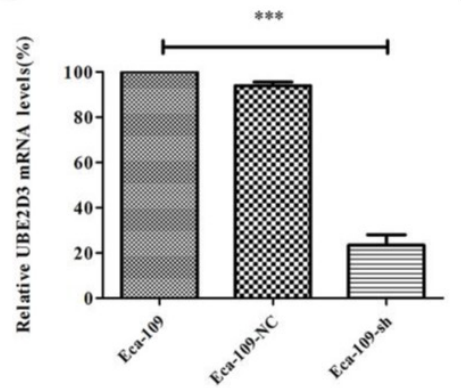

B

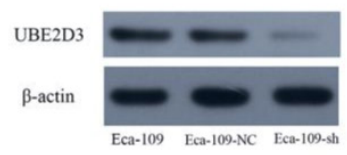

C

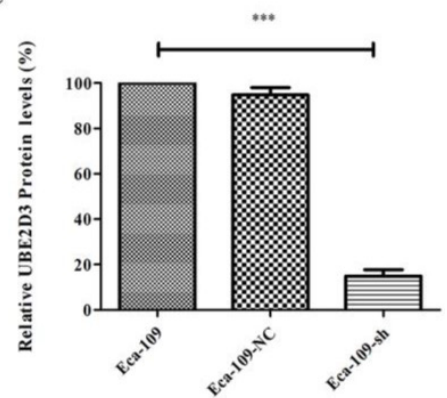

Figure 1. shRNA against UBE2D3 decreases UBE2D3 mRNA and protein levels. (a) Real-time PCR detected the relative UBE2D3 mRNA levels in Eca-109, Eca-109-NC and Eca-109-sh cells. (b) The image of western blot detected the relative UBE2D3 protein levels in Eca-109, Eca-109-NC and Eca-109-sh cells. (c) The bands density analysis of western blot detected the relative UBE2D3 protein levels in Eca-109, Eca-109-NC and Eca-109-sh cells. The bar graph shows the mean \pm standard deviation (SD) values of three independent experiments. ***P<0.0005 Eca-109-sh vs. Eca-109-NC group. Eca-109-NC group revealed no apparent differences compared with the Eca-109 group. 
Table 1. Radiobiological parameters in the different groups. D0, the incremental dose required for reducing the fraction of colonies to $37 \%$, indicative of single-event killing; $\mathrm{Dq}$, quasi-threshold dose; SF2, the survival fraction after $2 \mathrm{~Gy}$ radiation exposure. ${ }^{*} \mathrm{P}<0.05$.

\begin{tabular}{llll}
\hline Cell line & D0 & Dq & SF2 \\
\hline Eca-109-NC & $1.964 \pm 0.315$ & $0.256 \pm 0.313$ & $0.3863 \pm 0.0103$ \\
Eca-109-sh & $3.253 \pm 0.190^{*}$ & $0.929 \pm 0.460^{*}$ & $0.6244 \pm 0.0069^{*}$ \\
\hline
\end{tabular}

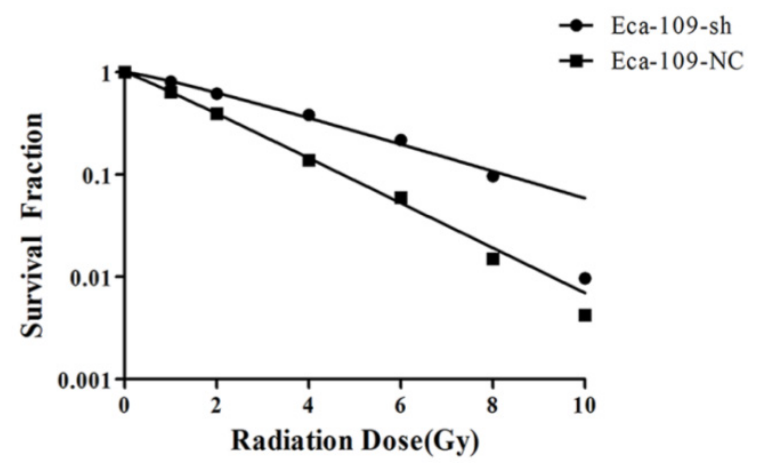

Figure 2. UBE2D3 knockdown confers esophageal cancer cells to radioresistance. Each group was irradiated with graded doses of $0,1,2,4,6$, $8,10 \mathrm{~Gy}$. After 14 days of incubation, the colonies were fixed and stained. Those colonies containing more than 50 cells were scored as viable colonies. These data were fit into the sing-hit multi-target model, and survival curves for each group were demonstrated using GraphPad prism 5.0 software.

\section{UBE2D3 downregulation enhances telomeric stability}

Telomeric stability is closely associated with radioresistance. Telomere maintenance is defined by telomere length and telomerase activity. Thus, to investigate the relationship between UBE2D3 and telomere stability, real-time PCR and TeloTAGGG Telomerase PCR ELISA were used to measure telomere length and telomerase activity, respectively, in Eca-109-NC and Eca-109-sh cell lines. UBE2D3 downregulation significantly enhanced telomere length and telomerase activity (Figure 3). The telomerase activity of Eca-109, Eca-109-NC, and

\section{A}

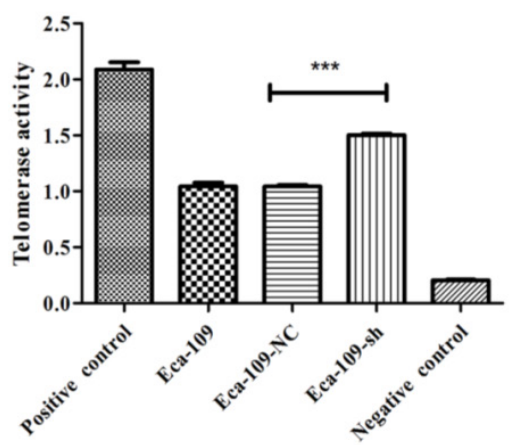

Eca-109-sh cells was $1.04 \pm 0.13,1.05 \pm 0.49$, and $1.50 \pm$ 0.06, respectively. The Eca-109 and Eca-109-NC groups showed no significant change. However, telomerase activity in the Eca-109-sh group was significantly higher than that in the Eca-109-NC group (Figure 3A, $\mathrm{P}<0.5$ ). Telomerase activity of the negative control was not more than 0.25 and that of the positive control was not less than 1.5 (Figure 3A), showing that telomerase activity could be successfully detected. The average telomere length in Eca-109-sh cells was $2.86 \pm 0.10$ fold higher than that in Eca-109-NC cells (Figure 3B). These results indicate that UBE2D3 expression decreases telomeric stability.

\section{UBE2D3 downregulation decreases the proportion of cells in the G2/M phase, but increases the proportion of cells in the $S$ phase and radiation-induced $G 2 / M$ phase arrest in Eca-109 cells}

Cell cycle is one of the key factors affecting radioresistance. To investigate the UBE2D3-mediated effect on the cell cycle, flow cytometry was performed to analyze the effect of UBE2D3 knockdown on the cell cycle. As shown in Figure 4A and 4B, compared to negative control cells, UBE2D3 depletion had no significant effect on the proportion of cells in the G1 phase, but it significantly affected the number of cells in the G2/M phase and S phase.

Eighteen hours after exposure to 6 Gy IR, the G2/M arrest reached a peak in both Eca-109-NC and Eca-109-sh cells, but the number of Eca-109-sh cells arrested in the G2/M phase was higher. More importantly, the response kinetic was different between these two cell types. In Eca-109-NC cells, the G2/M peak gradually decreased from $18 \mathrm{~h}$ after IR and returned to basal levels at approximately $30 \mathrm{~h}$. However, the G2/M peak in Eca-109-sh cells did not decrease and was maintained at a high level until $42 \mathrm{~h}$ after IR. These results suggest that UBE2D3 downregulation in Eca-109 cells prolonged G2/M arrest after IR exposure (Figure 4A and 4C).

B

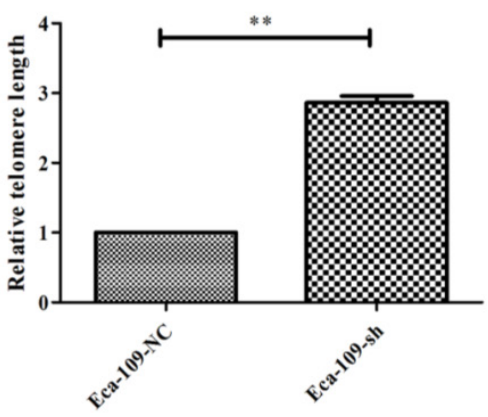

Figure 3. UBE2D3 downregulation enhances telomeric stability. (a) PCR-ELISA was performed to detect telomerase activity. Compared with Eca-109-NC group, Eca-109-sh group showed significantly higher telomerase activity. $* * * P<0.0001$. (b) Real-time PCR was determined to analyze the telomere length in Eca-109-NC and Eca-109-sh groups, respectively. Compared with Eca-109-NC group, Eca-109-sh group showed significantly longer telomere length. **P<0.005. 
A

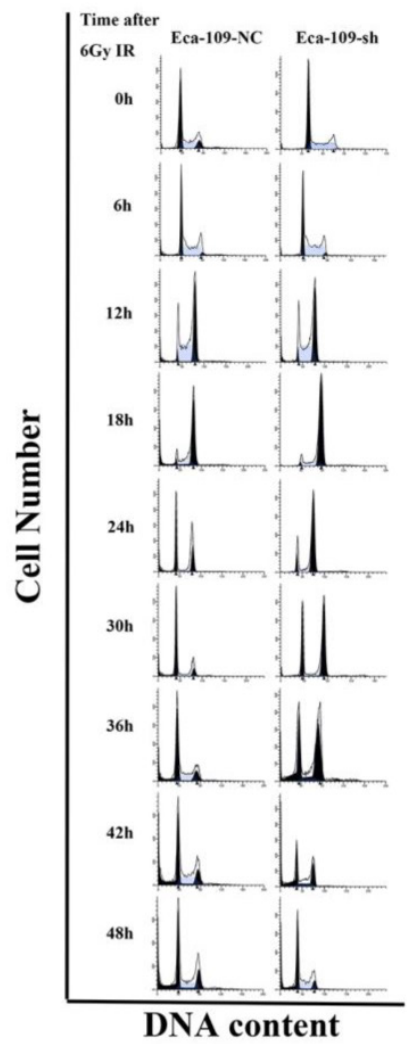

B

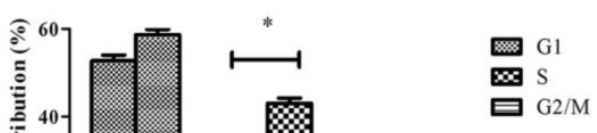

C

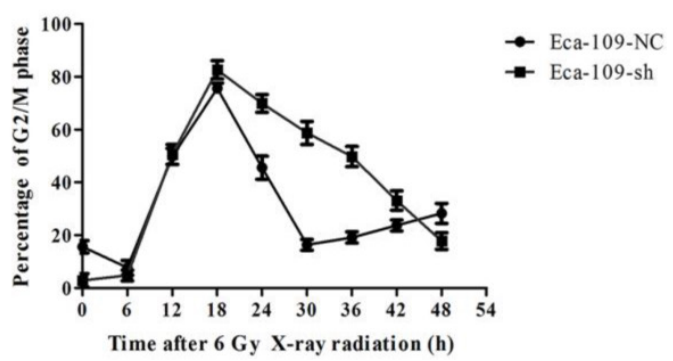

Figure 4. Effects of UBE2D3 downregulation on the cell cycle of Eca-109 cells by flow cytometry. (a) Eca-109-NC and Eca-109-sh cells were irradiated with 6Gy X-ray and recovered for indicated times. (b) The change of cell cycle phase distribution after UBE2D3 knockdown. *P<0.05. (c) The population of cells in G2/M phases over time in Eca-109-NC and Eca-109-sh cells.

A

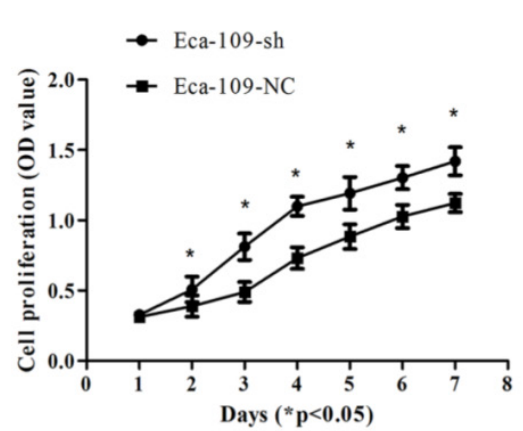

B

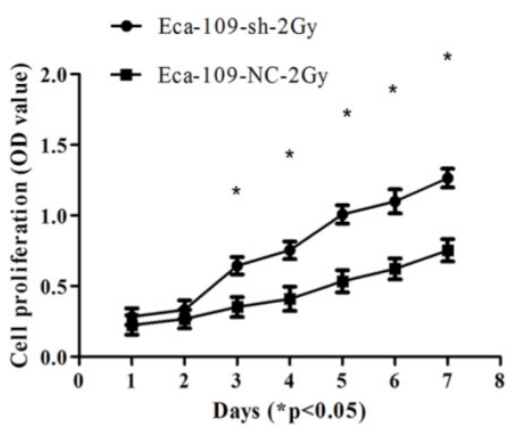

Figure 5. Proliferation of Eca-109 cells was determined by the cck-8 assay. (a) Eca-109-sh cells exhibited profoundly accelerated growth kinetics compared to Eca-109-NC cells exposed to 0Gy. *P<0.05. (b) Eca-109-sh cells exhibited profoundly accelerated growth kinetics compared to Eca-109-NC cells exposed to 2Gy. $* \mathrm{P}<0.05$.

\section{UBE2D3 is involved in controlling Eca-109 cell proliferation}

UBE2D3 downregulation promoted the proliferation of Eca-109 cells (Figure 5A). Additionally, after exposure to 2 Gy IR, the proliferation of Eca-109-sh cells was significantly higher than that of Eca-109-NC cells (Figure 5B) $(\mathrm{P}<$ 0.05).

\section{UBE2D3 downregulation decreases spontaneous and ionizing radiation-induced apoptosis}

Irradiation is known to cause DNA damage. If DNA damage repair is insufficient after irradiation, cells may undergo apoptosis and/or necrosis. Since UBE2D3 downregulation desensitized Eca-109 cells to irradiation, we further investigated the effects of UBE2D3 knockdown on cell apoptosis. The percentage of apoptotic cells was assessed using 
Annexin V-FITC staining, followed by flow cytometry. The percentage of apoptotic cells for each group was as follows: Eca-109-NC: $6.08 \pm 1.15 \%$, Eca-109-sh: $2.00 \pm 0.72 \%$, Eca-109-NC + IR: $14.3 \pm$ $0.95 \%$, and Eca-109-sh + IR: $3.77 \pm 1.56 \%$. The percentage of cells undergoing spontaneous or ionizing radiation-induced apoptosis was significantly decreased in Eca-109-sh cells compared to that in Eca-109-NC cells (Figure 6, P < 0.05). These results suggest that UBE2D3 knockdown reduces spontaneous and ionizing radiation-induced apoptosis in Eca-109 cells.

A
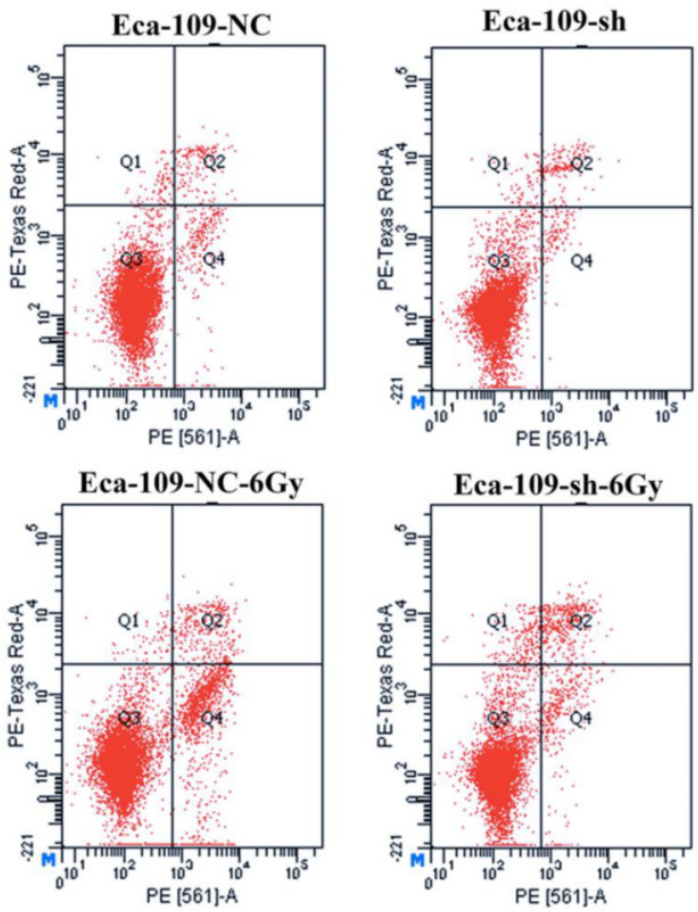

B

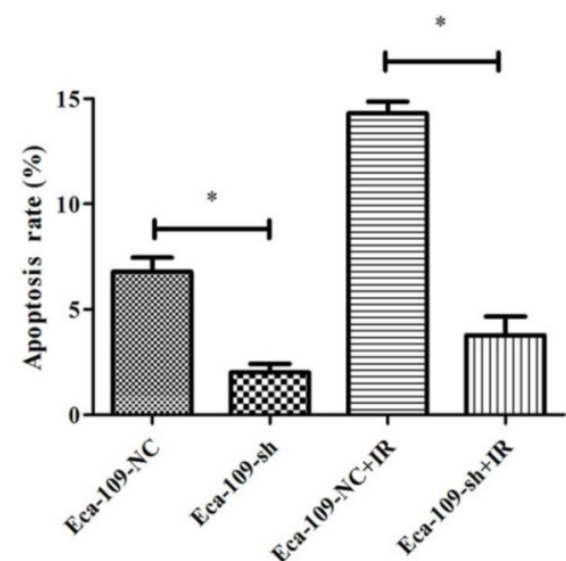

Figure 6. UBE2D3 downregulation decreases spontaneous and ionizing radiation-induced apoptosis. (a) The cell apoptoses of Eca-109-NC and Eca-109-sh cell lines were performed by flow cytometry prior to and post 6Gy irradiation. (b) Data are presented as means \pm SD from three independent experiments. $* \mathrm{P}<0.05$.
UBE2D3 downregulation reduces spontaneous DSB and accelerates the repair of DNA damage induced by IR

To investigate the effect of UBE2D3 on DNA damage repair, immunofluorescence was used to assess the changes in phospho- $\gamma \mathrm{H} 2 \mathrm{AX}$ after UBE2D3 knockdown. As shown in Figure 7, $\gamma \mathrm{H} 2 \mathrm{AX}$ foci significantly decreased in Eca-109-sh cells when compared with those in Eca-109-NC cells $(\mathrm{P}<0.05)$. One hour after $4 \mathrm{~Gy}$ irradiation, the number of $\mathrm{\gamma H} 2 \mathrm{AX}$ foci also significantly decreased in Eca-109-sh cells compared with that in Eca-109-NC cells $(P<0.05)$.

\section{Mechanisms involved in UBE2D3 downregulation-mediated changes in telomere homeostasis, cell cycle, cell apoptosis, and DNA damage repair}

To better understand UBE2D3-mediated effects on telomeres and telomerase, we determined the effect of UBE2D3 knockdown on the hTERT and shelterin protein complex. UBE2D3 knockdown significantly increased protein levels of hTERT, TRF1, TRF2, POT1, and RAP1, but had no effect on TPP1 and TIN2 protein levels (Figure 8A). Twelve hours after irradiation with 2 Gy and 4 Gy, respectively, TRF2 protein levels decreased in Eca-109-NC cells, but increased in Eca-109-sh cells. Moreover, these opposite effects on Eca-109-NC and Eca-109-sh cells were dose-dependent (Figure $8 \mathrm{C}$ ). These results indicate that UBE2D3 knockdown has protective effects against irradiation on telomeres.

To investigate the mechanisms involved in the changes mediated by UBE2D3 downregulation, the expressions of Bax and Bcl-2 proteins were assessed by western blot. The proportion of $\mathrm{Bax} / \mathrm{Bcl}-2$ was reduced in the absence of UBE2D3 (Figure 8B).

To determine the mechanisms involved in the DNA damage repair and cell cycle changes induced by UBE2D3 knockdown, the expression of DNA damage repair proteins (ataxia telangiectasia mutated, ATM; ataxia telangiectasia rad3-related, ATR; p-ATM; p-ATR; and $\gamma \mathrm{H} 2 \mathrm{AX}$ ) and cell cycle check point proteins (cyclin D1, CDC25A, CDC25C, and Chk1) were assessed by western blot. UBE2D3 knockdown induced a significant increase in ATM, ATR, p-ATM, cyclin D1, and Chk1 expression, while it significantly reduced $\gamma \mathrm{H} 2 \mathrm{AX}, \mathrm{CDC} 25 \mathrm{~A}$, and $\mathrm{CDC} 25 \mathrm{C}$ protein expression (Figure 8B). However, no effect was observed on p-ATR protein levels (Figure 8B). When exposed to radiation, the protein levels of ATM and ATR were further increased when UBE2D3 was downregulated. The increases in ATM and ATR protein levels were greater after $4 \mathrm{~Gy}$ irradiation than after $2 \mathrm{~Gy}$ exposure. However, CDC25C, and $\gamma \mathrm{H} 2 \mathrm{AX}$ 
protein levels, decreased when UBE2D3 was downregulated after irradiation (Figure 8C).

\section{Discussion}

In the current study, we demonstrated for the first time that the ubiquitin-conjugating enzyme
UBE2D3 is involved in the regulation of radioresistance and telomere maintenance in Eca-109 cells.

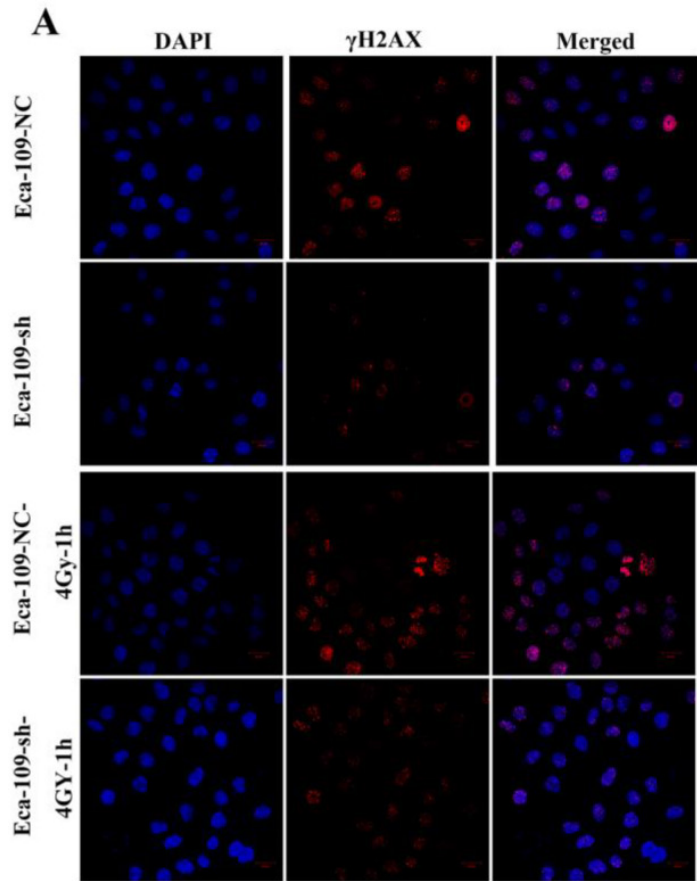

B

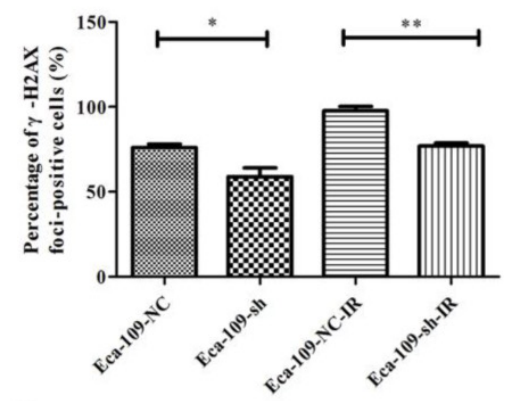

C

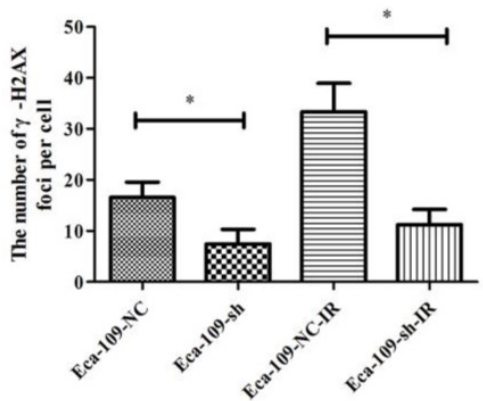

Figure 7. UBE2D3 knockdown decreases the YH2AX-mediated repair of DSBs. (a) Confocal microscopy records the images in 4 groups as follows: Eca-109-NC, Eca-109-sh, Eca-109-NC-4Gy-1h and Eca-109-sh-4Gy-1h. (b) The percentages of YH2AX foci-positive cells in these four groups were obtained by analysing 100 randomly selected cells in each group. $* P<0.05$, $* * P<0.005$. (c) The numbers of $\mathrm{H} 2 \mathrm{AX}$ foci per cell in these four groups were obtained by analysing 100 randomly selected cells in each group. ${ }^{*} \mathrm{P}<0.05$.

A

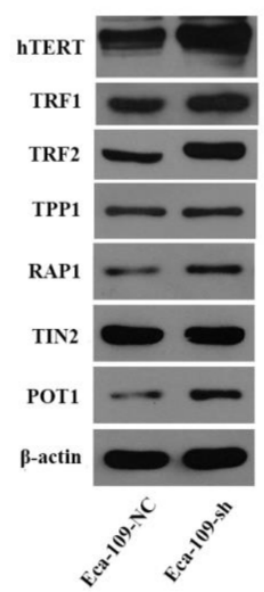

B

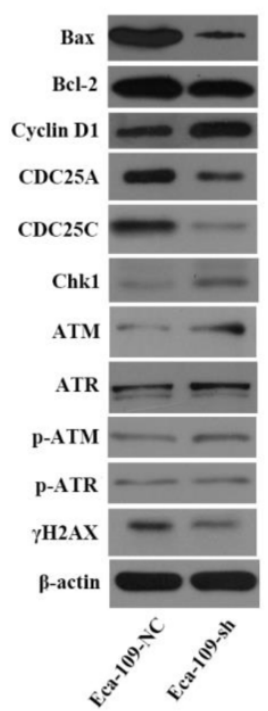

C

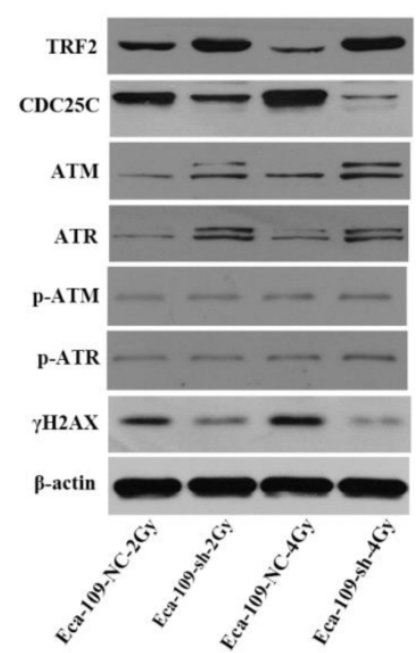

Figure 8. Mechanisms involved in UBE2D3 downregulation-mediated changes in telomere homeostasis, cell cycle, cell apoptosis, and DNA damage repair. (a) The effect of knockdown UBE2D3 on telomere homeostasis. (b) The effects of knockdown UBE2D3 on cell cycle, cell apoptosis and double-strand breaks proteins. (c) The effects of knockdown UBE2D3 on the expressions of TRF2, CDC25C, ATM, ATR, p-ATM, p-ATR and YH2AX, $24 \mathrm{~h}$ after 2Gy, 4Gy irradiation. 
Ubiquitylation modification, which is mediated by the ubiquitin/proteasome system (UPS), plays an important role in DNA damage response activated by DNA DSBs as well as some other posttranslational protein modifications such as phosphorylation, acetylation and methylation [19]. Moreover, recent studies have shown that E2 enzyme family members participate in DNA damage repair and affect radioresistance [8, 20] [21]. Our previous study indicated that UBE2D3, an ubiquitin conjugating enzyme, plays a role in radioresistance in human breast cancer [8]; however, until now, it has been unknown whether this role of UBE2D3 in radioresistance is limited to breast cancer. In this study, we used the clonogenic assay to assess the radioresistance in an esophageal cancer cell line, Eca-109, after UBE2D3 knockdown, and demonstrated that UBE2D3 knockdown induced radioresistance.

Our previous study demonstrated that the expression of UBE2D3 was negatively related to human telomerase reverse transcriptase (hTERT) [8]. hTERT is the most important factor to increasetelomerase activity, which in turn is a key regulator of radioresistance [22]. Thus, to determine the mechanisms by which UBE2D3 regulates radioresistance in Eca-109 cells, we first determined hTERT expression, telomerase activity and telomere length. The results showed that UBE2D3 knockdown raised hTERT protein expression, enhanced telomerase activity, and increased telomere length. In vitro studies previously showed that the inhibition of telomerase activity decreased DNA damage repair, shortened telomeres, and decreased radioresistance [23] [22]. Telomere length is positively related to radioresistance [24]. In addition, telomerase activity and telomere length are positively related to telomere homeostasis, leading to a state in which the structural integrity and function of the telomere are maintained $[22,24]$. Moreover, telomere homeostasis is positively related to radioresistance [3]. Thus, our study suggests that radioresistance induced by UBE2D3 knockdown is related to the enhancement of telomere homeostasis resulting from increases in telomerase activity and telomere length.

To verify this hypothesis, we assessed the expression of telomere shelterin proteins which play a protective role and are positively associated with the state of telomere homeostasis [25, 26], and found that UBE2D3 knockdown increased the expressions of TRF1, TRF2, POT1 and RAP1, but did not affect the expressions of TPP1 and TIN2. These results suggest that downregulation of UBE2D3 promotes the maintenance of telomere homeostasis. As TRF2 is a key protein that binds to the double strand of the telomere [27], we chose it for further study of telomere homeostasis after 2 Gy or 4 Gy irradiation and determined that UBE2D3 knockdown increased TRF2 expression in a dose dependent manner. These results suggest that UBE2D3 knockdown regulates radioresistance, probably via enhancing telomere protection.

Classical radiation biology suggests that the changes in the cell cycle distribution are one of the key factors regulating radioresistance. The G1 phase and early $S$ phase are the most radioresistant phases of the cell cycle, while the $\mathrm{G} 2 / \mathrm{M}$ phase is the most radiosensitive phase [4]. Changes in the expression of cell cycle checkpoint proteins result in changes in the cell cycle distribution. Previous studies indicated that cyclin D1 promotes a shift from the G1 to $S$ phase, and CDC25A accelerates the $S$ to $G 2$ phase transition [28]. Recently, some studies revealed that ubiquitylation plays an important role in the regulation of cell cycle distribution [29] [30]. Cyclin D1 is a downstream target of UBE2D3 [31]. Thus, the change in the cell cycle distribution after UBE2D3 knockdown might be another mechanism underlying the induction of radioresistance. In the present study, UBE2D3 knockdown had no significant effect on the proportion of cells in the G1 phase, but significantly increased the number of cells in the $S$ phase, whereas it reduced the number of cells in G2/M phase arrest. To study the mechanisms involved in the changes observed in cell cycle distribution, changes in the levels of cell cycle check point proteins after UBE2D3 knockdown were determined. Cyclin D1 was overexpressed, and CDC25A expression was reduced after UBE2D3 knockdown. Therefore, this study indicates that UBE2D3 depletion leads to an increase in the $S$ phase, but a decrease in the G2/M phase. Our study thus indicates that changes in cell cycle distribution might be a factor underlying radioresistance after UBE2D3 knockdown.

When radiation-induced DNA damage occurs, ATM and ATR protein kinases are activated to induce cell cycle arrest [32]. Phosphorylation of ATM can activate Chk1 by phosphorylation on S345 [33]. CDC25C plays a role in the $\mathrm{G} 2$ to $\mathrm{M}$ phase transition [28]. Chk1 phosphorylation inhibits CDC25C activity and leads to G2/M arrest [34]. To confirm that UBE2D3 knockdown-induced cell cycle changes are involved in radioresistance, the cell cycle distribution was assessed at different time points after 6Gy irradiation. UBE2D3 knockdown combined with 6Gy irradiation led to prolonged G2/M arrest. In addition, UBE2D3 knockdown increased the expressions of shelterins, ATM and ATR, but reduced the expressions of Chk1 and CDC25C in cells treated with or without radiation exposure. Therefore, this study 
indicates that UBE2D3 knockdown combined with radiation could promote the transformation of Chk1 into phosphorylation of Chk1 via increasing the activation of ATM and ATR. These processes lead to decreased chk1 levels, thereby increasing the inhibition of CDC25C, which leads to prolonged $\mathrm{G} 2 / \mathrm{M}$ arrest. Thus, prolonged G2/M arrest induced by knockdown of UBE2D3 may be mediated via the ATM/ATR-Chk1-CDC25C signaling pathway.

The proportion of proliferating cells that are sensitive to radiation is negatively related to radioresistance [6]. As the radiation induced cell apoptosis results from inadequate DNA damage repair after irradiation, the disabling of cell apoptosis may result in radioresistance [5]. Furthermore, previous studies have shown that the expressions of TRF1 and TRF2 were negatively related to apoptosis [35], while cyclin D1 and hTERT were positively associated with proliferation [36]. Thus, UBE2D3 knockdown might regulate cell proliferation and cell apoptosis. Our study showed that UBE2D3 knockdown accelerated the cell proliferation, while reduced the proportion of radiosensitive proliferating cells and the proportion of cells undergoing spontaneous and radiation-induced apoptosis. Bax functions as a pro-apoptotic protein, whereas Bcl-2 functions as an anti-apoptotic protein. The Bax/Bcl-2 ratio is an indicator of cell apoptosis. A recent report indicated that the reduction of telomerase activity is related to an increase in the Bax/Bcl-2 ratio [37]. Hence, the increased proliferation induced by UBE2D3 depletion might be mediated by increasing hTERT and cyclin D1 protein levels, whereas the reduced spontaneous and radiation-induced apoptosis might result from the decrease in Bax/Bcl-2 ratio and the increases in the telomerase activity and the protein levels of TRF1 and TRF2. However, the exact mechanisms that underlie these phenomena require further study.

As a histone $\mathrm{H} 2 \mathrm{~A}$ variant, $\mathrm{H} 2 \mathrm{AX}$ plays an essential role in the cellular response to DNA DSBs. H2AX senses DSBs through rapid serine 139 phosphorylation and forms phospho- $\gamma \mathrm{H} 2 \mathrm{AX}$ foci with various proteins [38]. In cells with different sensitivities to IR-induced DSBs, $\gamma \mathrm{H} 2 \mathrm{AX}$ selectively recruits specific proteins to determine cell fate [39]. Thus, the number of $\gamma \mathrm{H} 2 \mathrm{AX}$ foci is a representation of DNA damage and the cell's capacity for DNA damage repair. In this study, the number of DNA damage foci decreased and the repair kinetics of total DSB increased after UBE2D3 knockdown, indicating that UBE2D3 knockdown enhanced DNA damage repair ability in esophageal cancer cells.

In conclusion, our results demonstrate that UBE2D3 downregulation promotes telomere maintenance and enhances radioresistance in esophageal cancer cells. Therefore, our study indicates for the first time that UBE2D3 may be a promising target to enhance the effects of radiotherapy and maintain telomere structural integrity and functional stability in esophageal cancer cells. Moreover, UBE2D3 overexpression might be useful to improve the success of radiation therapy, a hypothesis currently under investigation in our laboratory.

\section{Competing Interests}

The authors have declared that no competing interest exists.

\section{References}

1. Minsky BD, Pajak TF, Ginsberg RJ, Pisansky TM, Martenson J, Komaki R, et al. INT 0123 (Radiation Therapy Oncology Group 94-05) phase III trial of combined-modality therapy for esophageal cancer: high-dose versus standard-dose radiation therapy. Journal of clinical oncology : official journal of the American Society of Clinical Oncology. 2002; 20: 1167-74.

2. Pal I, Dey KK, Chaurasia M, Parida S, Das S, Rajesh Y, et al. Cooperative effect of BI-69A11 and celecoxib enhances radiosensitization by modulating DNA damage repair in colon carcinoma. Tumour biology : the journal of the International Society for Oncodevelopmental Biology and Medicine. 2015.

3. Ayouaz A, Raynaud C, Heride C, Revaud D, Sabatier L. Telomeres: hallmarks of radiosensitivity. Biochimie. 2008; 90: 60-72.

4. Pawlik TM, Keyomarsi K. Role of cell cycle in mediating sensitivity to radiotherapy. International journal of radiation oncology, biology, physics. 2004; 59: 928-42.

5. Eriksson D, Stigbrand T. Radiation-induced cell death mechanisms. Tumour biology : the journal of the International Society for Oncodevelopmental Biology and Medicine. 2010; 31: 363-72.

6. Prokopiou S, Moros EG, Poleszczuk J, Caudell J, Torres-Roca JF, Latifi K, et al. A proliferation saturation index to predict radiation response and personalize radiotherapy fractionation. Radiation oncology (London, England). 2015; 10: 159.

7. Tang Y, Geng Y, Luo J, Shen W, Zhu W, Meng C, et al. Downregulation of ubiquitin inhibits the proliferation and radioresistance of non-small cell lung cancer cells in vitro and in vivo. Scientific reports. 2015; 5: 9476.

8. Wang W, Yang L, Hu L, Li F, Ren L, Yu H, et al. Inhibition of UBE2D3 expression attenuates radiosensitivity of MCF-7 human breast cancer cells by increasing hTERT expression and activity. PloS one. 2013; 8: e64660.

9. Mittal MK, Singh K, Misra S, Chaudhuri G. SLUG-induced elevation of D1 cyclin in breast cancer cells through the inhibition of its ubiquitination. The Journal of biological chemistry. 2011; 286: 469-79.

10. Wu K, Kovacev J, Pan ZQ. Priming and extending: a UbcH5/Cdc34 E2 handoff mechanism for polyubiquitination on a SCF substrate. Molecular cell. 2010; 37: 784-96.

11. Saville MK, Sparks A, Xirodimas DP, Wardrop J, Stevenson LF, Bourdon JC, et al. Regulation of p53 by the ubiquitin-conjugating enzymes $\mathrm{UbcH} 5 \mathrm{~B} / \mathrm{C}$ in vivo. The Journal of biological chemistry. 2004; 279: 42169-81.

12. Okamoto Y, Ozaki T, Miyazaki K, Aoyama M, Miyazaki M, Nakagawara A. $\mathrm{UbcH} 10$ is the cancer-related E2 ubiquitin-conjugating enzyme. Cancer research. 2003; 63: 4167-73.

13. Polanowska J, Martin JS, Garcia-Muse T, Petalcorin MI, Boulton SJ. A conserved pathway to activate BRCA1-dependent ubiquitylation at DNA damage sites. The EMBO journal. 2006; 25: 2178-88.

14. Sato Y, Yamagata A, Goto-Ito S, Kubota K, Miyamoto R, Nakada S, et al. Molecular basis of Lys-63-linked polyubiquitination inhibition by the interaction between human deubiquitinating enzyme OTUB1 and ubiquitin-conjugating enzyme UBC13. The Journal of biological chemistry. 2012; 287: 25860-8

15. Wiener R, DiBello AT, Lombardi PM, Guzzo CM, Zhang X, Matunis MJ, et al E2 ubiquitin-conjugating enzymes regulate the deubiquitinating activity of OTUB1. Nature structural \& molecular biology. 2013; 20: 1033-9.

16. Li Y, Sun XX, Elferich J, Shinde U, David LL, Dai MS. Monoubiquitination is critical for ovarian tumor domain-containing ubiquitin aldehyde binding protein 1 (Otub1) to suppress $\mathrm{UbcH} 5$ enzyme and stabilize p53 protein. The Journal of biological chemistry. 2014; 289: 5097-108.

17. Guan GG, Wang WB, Lei BX, Wang QL, Wu L, Fu ZM, et al. UBE2D3 is a positive prognostic factor and is negatively correlated with hTERT expression in esophageal cancer. Oncology letters. 2015; 9: 1567-74.

18. Cawthon RM. Telomere measurement by quantitative PCR. Nucleic acids research. 2002; 30: e47.

19. Jacobs JJ. Fusing telomeres with RNF8. Nucleus. 2012; 3: 143-9.

20. Rawat A, Gopal G, Selvaluxmy G, Rajkumar T. Inhibition of ubiquitin conjugating enzyme UBE2C reduces proliferation and sensitizes breast cancer 
cells to radiation, doxorubicin, tamoxifen and letrozole. Cellular oncology. 2013; 36: 459-67.

21. Zhang P, Wang L, Rodriguez-Aguayo C, Yuan Y, Debeb BG, Chen D, et al. miR-205 acts as a tumour radiosensitizer by targeting ZEB1 and Ubc13. Nature communications. 2014; 5: 5671.

22. Ji XM, Xie $\mathrm{CH}$, Fang MH, Zhou FX, Zhang WJ, Zhang MS, et al. Efficient inhibition of human telomerase activity by antisense oligonucleotides sensitizes cancer cells to radiotherapy. Acta pharmacologica Sinica. 2006; 27: $1185-91$

23. Zhou FX, Liao ZK, Dai J, Xiong J, Xie CH, Luo ZG, et al. Radiosensitization effect of zidovudine on human malignant glioma cells. Biochemical and biophysical research communications. 2007; 354: 351-6.

24. Zhong YH, Liao ZK, Zhou FX, Xie CH, Xiao CY, Pan DF, et al. Telomere length inversely correlates with radiosensitivity in human carcinoma cells with the same tissue background. Biochemical and biophysical research communications. 2008; 367: 84-9.

25. Martinez P, Blasco MA. Telomeric and extra-telomeric roles for telomerase and the telomere-binding proteins. Nature reviews Cancer. 2011; 11: 161-76.

26. Choi KH, Farrell AS, Lakamp AS, Ouellette MM. Characterization of the DNA binding specificity of Shelterin complexes. Nucleic acids research. 2011; 39: 9206-23.

27. Yang $\mathrm{L}$, Wang $\mathrm{W}, \mathrm{Hu} \mathrm{L}$, Yang $\mathrm{X}$, Zhong J, Li Z, et al. Telomere-binding protein TPP1 modulates telomere homeostasis and confers radioresistance to human colorectal cancer cells. PloS one. 2013; 8: e81034.

28. Pozo-Molina G, Ponciano-Gomez A, Rivera-Gonzalez GC, Hernandez-Zavala A, Garrido E. Arsenic-induced S phase cell cycle lengthening is associated with ROS generation, p53 signaling and CDC25A expression. Chemico-biological interactions. 2015; 238: 170-9.

29. Halaby MJ, Hakem R, Hakem A. Pirh2: an E3 ligase with central roles in the regulation of cell cycle, DNA damage response, and differentiation. Cell cycle (Georgetown, Tex). 2013; 12: 2733-7.

30. Craney A, Rape M. Dynamic regulation of ubiquitin-dependent cell cycle control. Current opinion in cell biology. 2013; 25: 704-10.

31. Hattori $\mathrm{H}$, Zhang $\mathrm{X}$, Jia $\mathrm{Y}$, Subramanian $\mathrm{KK}$, Jo $\mathrm{H}$, Loison F, et al. RNAi screen identifies UBE2D3 as a mediator of all-trans retinoic acid-induced cell growth arrest in human acute promyelocytic NB4 cells. Blood. 2007; 110: 640-50.

32. Hurley PJ, Bunz F. ATM and ATR: components of an integrated circuit. Cell cycle (Georgetown, Tex). 2007; 6: 414-7.

33. Borst GR, McLaughlin M, Kyula JN, Neijenhuis S, Khan A, Good J, et al. Targeted radiosensitization by the Chk1 inhibitor SAR-020106. International journal of radiation oncology, biology, physics. 2013; 85: 1110-8.

34. Koo D. Chronic fatigue syndrome. A critical appraisal of the role of Epstein-Barr virus. The Western journal of medicine. 1989; 150: 590-6.

35. Pal D, Sharma U, Singh SK, Kakkar N, Prasad R. Over-expression of telomere binding factors (TRF1 \& TRF2) in renal cell carcinoma and their inhibition by using SiRNA induce apoptosis, reduce cell proliferation and migration invitro. PloS one. 2015; 10: e0115651.

36. Jiang W, Kahn SM, Zhou P, Zhang YJ, Cacace AM, Infante AS, et al. Overexpression of cyclin D1 in rat fibroblasts causes abnormalities in growth control, cell cycle progression and gene expression. Oncogene. 1993; 8: $3447-57$

37. Vafaiyan Z, Gharaei R, Asadi J. The correlation between telomerase activity and $\mathrm{Bax} / \mathrm{Bcl}-2$ ratio in valproic acid-treated MCF-7 breast cancer cell line. Iranian journal of basic medical sciences. 2015; 18: 700-4.

38. Stucki M, Jackson SP. gammaH2AX and MDC1: anchoring the DNA-damage-response machinery to broken chromosomes. DNA repair. 2006; 5: 534-43.

39. Keogh MC, Kim JA, Downey M, Fillingham J, Chowdhury D, Harrison JC, et al. A phosphatase complex that dephosphorylates gammaH2AX regulates DNA damage checkpoint recovery. Nature. 2006; 439: 497-501. 